\title{
Treatment of retained encrusted ureteral Double-J stent
}

\author{
Ibrahim Alnadhari ${ }^{1,2}$, Mohammed Ahmed Alwan ${ }^{1}$, Morshed Ali Salah ${ }^{1,2}$, Abdulelah M. Ghilan ${ }^{1}$ \\ ${ }^{1}$ Urology and Nephrology Center, at Al-Thawra Modern General and Teaching Hospital, Sana'a, Yemen; \\ ${ }^{2}$ Department of Urology, Al Wakra Hospital, Hamad Medical Corporation, Al Wakra, Qatar.
}

\begin{abstract}
Summary Objectives: We conducted this study to evaluate patients with retained encrusted ureteral stents, identify the predisposing factors and present our experience in the management of such challenging problem. Materials and Methods: This prospective study was carried out in the period from May 2007 to February 2011 at the Urology and Nephrology Center, Al-Thawra General Hospital, Sana'a, Yemen. 40 patients with retained encrusted ureteric stents were treated at our center. All patients were initially evaluated with a radiographic imaging for assessment of stent encrustation and stone burden. Treatment decisions were based on the site and severity of encrustations in the renal pelvis, ureter and bladder and on our technical situation and availability of instrumentations. Multi-modal approaches ranging from extracorporeal shock wave lithotripsy (ESWL) to endourological and open urologic procedures were used to achieve stent removal. Results: A total of 90 urological procedures were performed to render all 40 patients stent and stone free. The average duration of stent remained indwelling was 24.2 months (range 4 months -16 years). All patients were managed either by minimally or more invasive multi-modal endourological approaches. For upper coil encrustation percutaneous nephrolithotripsy was performed in eight patients, pyelolithotomy in two patients and ESWL in three patients. Encrustation of the body was treated initially by ESWL, followed by retrograde ureteroscopic manipulation in 12 patients. Lower coil encrustation was successfully managed by cystolitholapaxy in seven patients and one patient required cystolithotomy. Cystolithotomy, pyelolithotomy and ureterolithotomy were carried out in two patients. Two patients who had large burden bladder and kidney stones with loss of kidney function underwent nephrectomy and cystolithotomy. Conclusions: The retrieval of severely encrusted retained ureteral stent and its associated stone burden poses a real management challenge for urologists due to the need for multimodal procedures and the lack of standardized treatment plan.
\end{abstract}

KEY WORDS: Ureteral stent; Double-J stent; Encrustation.

Submitted 25 July 2018; Accepted 13 August 2018

\section{INTRODUCTION}

The use of indwelling ureteral stents has become an integral part of many urological procedures. They provide free drainage from upper urinary tract to the bladder in cases of renal and ureteral obstruction secondary to a variety of intrinsic or extrinsic factors such as calculi, strictures, congenital anomalies, pelvic malignancies, retro-peritoneal tumors, and fibrosis (1-4). Common indications for stent placement include the prevention or relief of obstruction prior to Extracorporal Shock Wave Lithotripsy (ESWL) and after ureteroscopy (URS) $(5,6)$. They are also placed after iatrogenic injuries of the ureter and for the purpose of easy identification and protection of the ureter during complex abdominal procedure (7). Since Zimskind et al. first described the original use of endoscopically inserted indwelling ureteral stent in 1967 (8),various modifications on stent design have been made allowing for easier manipulation $(1,6)$. Modern ureteral stents are generally designed in a double-pigtail or double-J (JJ) configuration $(3,9)$. The ideal stents are those which are easy to insert and remove, radiologically-opaque, having good flow characteristics, biologically inert and chemically stable in urinary tract, and the stent biomaterial should resist encrustation, prevent infection and be widely avail-able at a reasonable cost (3).

Unfortunately, no stent biomaterial is currently available that meets all of these criteria.

A variety of materials including synthetic polymeric compounds (polyurethane/polyethylene) and silicon are available with various biocompatibility and biodurability $(3,6)$. Despite advances in stent deigns and materials, problems related to indwelling ureteral stent use, such as infection, encrustation, stone formation, occlusion, migration and breakage continue to occur $(1,5,10,11)$. Retained ureteral stents especially those that are encrusted can be a challenging problem that may lead to serious complications of obstruction, infection and renal impairment if not managed properly $(1,2,6,12)$. The problem of retained stents occurs due to variety of causes. Illiteracy and non-compliance of the patient together with poor communication between patients and physicians are the main contributing causes for delay in timely removal of stents $(2,13)$.

We conducted this study to evaluate patients with retained encrusted indwelling ureteral stents, identify the predisposing factors and present our experience in the management of such challenging problem.

\section{Materials AND MEthods}

A prospective, observational and cohort study was carried out in the period from May 2007 to February 2011 at the Urology and Nephrology Center, Al-Thawra General and Teaching Hospital, the main referral hospital in the country, Sana'a, Yemen. 40 patients (30 males and 10 females) who were presented with retained encrusted 
ureteric stents and managed at our hospital during this period. All patients with a retained stent which was defined as "one which could not be removed cystoscopically at the first attempt and required other auxiliary measures or surgical procedure for removal", were included in the study. Patients with no visible encrustation on their stents, in whom the stents were easily removed cystoscopically at first attempt, were excluded from the study, no matter how long was the duration of missing.

The indications of initial stent placement, the duration of stent in the urinary system, and the reasons for delayed removal were all reviewed in the history. All patients were initially evaluated with a plain radiography (KUB), abdominal ultrasonography and intravenous urography (IVU) for assessment of stent encrustation and associated stone burden. Intravenous urography and in some cases, renal isotope scan were also obtained to document the degree of renal function loss. Laboratory tests such as complete blood count, urea and creatinine measurements and urine culture and sensitivity tests were routinely performed.

Treatment decisions were based on the site and severity of encrustations in the renal pelvis, ureter and bladder and on our technical situation and availability of instrumentations. Patients were counseled about the benefits, risks and possible complications including sepsis, loss of renal function, injury to surrounding organs and the possible need for further interventions. Cystolithotripsy and cystolithotomy were required to treat the distal components of stent. ESWL, ureterorenoscopic (URS) manipulations, and ureterolithotomy were used to treat the ureteric part of the stent. ESWL, percutaneous nephrolithotripsy (PCNL), and pyelolithotomy were performed to treat the upper coil of the stent, and nephrectomy for removal of non-functioning kidney. All of these procedures were carried out under general anesthesia, except ESWL sessions which were performed under parenteral analgesia. Post-treatment control KUB films and/or ab-domino-pelvic ultrasonography were routinely performed for our patients.

\section{RESULTS}

Forty patients with retained ureteral stents were managed in our center during the period of the study. Patients' ages ranged from 4 to 70 years (mean 30 years), 30 patients were males and 10 were females. The average duration of stent remained indwelling was 24.2 months (range 4 months- 16 years). All stents (except two) were placed in the capital Sana'a. One of the remaining two was placed in Saudi Arabia and the other in Ibb city. In 22 patients (55\%), the stent side was the right while in the remainder 18 (45\%), the side was the left.

The most common reasons for stent missing, as shown in Table 1, were poor compliance of the patients (patients ignored or forgot physicians' advice regarding its timely removal) and seen in $47.5 \%$ of cases, followed by inability to return back to hospital due to financial reasons (30\%), and delayed referral after ESWL to endourology department for stent replacement or removal in the optimal time $(12.5 \%)$. In $10 \%$ of patients, the reason was poor communication between patients and physicians
Table 1.

Baseline characteristic and clinical features of the study population.

\begin{tabular}{|c|c|c|}
\hline Number of patients $n^{\circ}$ & no (\%) & 40 \\
\hline$\overline{\text { Sex (male/female) }}$ & (Male/female) & $30 / 10(75 / 25 \%)$ \\
\hline Age & Mean (range) & $30(4-70)$ years \\
\hline Residency & $\begin{array}{l}\text { Sana'a } \\
\text { Others }\end{array}$ & $\begin{array}{l}13(32.5 \%) \\
27(67.5 \%)\end{array}$ \\
\hline Cause of missing stents & $\begin{array}{c}\text { Poor compliance } \\
\text { Inability to return back } \\
\text { Delay in referral } \\
\text { Poor communication }\end{array}$ & $\begin{array}{c}19(47.5 \%) \\
12(30 \%) \\
5(12.5 \%) \\
4(10 \%)\end{array}$ \\
\hline $\begin{array}{l}\text { Indication for initial stent } \\
\text { placement }\end{array}$ & $\begin{array}{l}\text { Relieve of obstruction } \\
\text { Intra-operative } \\
\text { Before ESWL }{ }^{\mathrm{a}}\end{array}$ & $\begin{array}{c}16(40 \%) \\
15(37.5 \%) \\
9(22.5 \%)\end{array}$ \\
\hline Site of maximum encrustation & $\begin{array}{l}\text { Kidney (upper coil) } \\
\text { Kidney \& ureter } \\
\text { Ureter (body) } \\
\text { Bladder (lower coil) } \\
\text { Kidney \& bladder } \\
\text { Totally encrusted } \\
\text { Not visible }\end{array}$ & $\begin{array}{c}6(15 \%) \\
4(10 \%) \\
15(37.5 \%) \\
6(15 \%) \\
4(10 \%) \\
2(5 \%) \\
3(7.5 \%)\end{array}$ \\
\hline Executed therapy & $\begin{array}{c}\text { ESWL } \\
\text { URS }^{b} \\
\text { Cystolitholapaxy } \\
\text { PCNL }^{c} \\
\text { Cystolithotomy } \\
\text { Pyelolithotomy } \\
\text { Ureterolithotomy } \\
\text { Nephrectomy }\end{array}$ & $\begin{array}{c}50(55.5 \%) \\
12(13.3 \%) \\
7(7.7 \%) \\
8(8.8 \%) \\
5(5.5 \%) \\
4(4.4 \%) \\
2(2.2 \%) \\
2(2.2 \%)\end{array}$ \\
\hline
\end{tabular}

(the patients did not know about the presence of stent and/or the need for its timely removal). The main initial indications of ureteral stenting and the sites of maximum encrustation are shown in Table 1.

Combinations of ESWL, endourologic and open urologic procedures were required to facilitate removal of retained stent and associated stones. A total of 90 urologic procedures were performed to render all 40 patients stent free. Averages of 2.25 urologic procedures per patients (range 1-10) were performed during either single or multiple anesthetic sessions as shown in Table 1. In 25 out of 40 patients, it was possible to have patients cleared from their retained stents and stone burden in a single anesthetic session. We began by careful evaluation of plain KUB for the presence of encrustation on the proximal coil, body and distal coil of the stent. In cases of no visible encrustation, cystoscopy and trial of stent extraction by gentle traction under fluoroscopic control was applied. If it was removed easily, then we did not include the case in our study despite the duration of missing. If we found difficulties in stent extraction, no force was applied to avoid damaging the ureter and the case was considered as a retained stent. ESWL was our first choice for cases of retained stent without visible encrustation or with minor encrustation especially on the proximal coil and body. We had three patients with retained stent without clear calcifications in whom ESWL to the proximal coil was carried out and then the stent was removed easily. In cases of visible encrustations 
on plain KUB, no attempts at direct removal were done, but we proceeded according to the site and severity of encrustations. If there were linear encrustations on the proximal coil or body, we performed ESWL to the site of encrustation and then trial of removal of stent by gentle traction without force. For linear or minor encrustation on the distal coil, we crushed them with forceps or lithotripter to fragment them and remove the fragments. Pneumatic lithotripter was used whenever needed. When these less invasive measures failed or if the encrustations were severe, then we manage our patients in retrograde fashion, starting with the bladder component, then the ureter and kidney. Cystolithotripsy was our first option for bladder component of encrustations. In children, we did cystolithotomy in two patients to remove bladder encrustations because of large stone burden and to avoid repeated urethral instrumentation and the risk of urethral injury in children, especially males. Cystolithotomy was also performed for three adults who had large bulky bladder stone around the distal coil. For ureteral components, we mostly started by preliminary ESWL to fragment the encrustations around the stent and then we passed a small $(7 \mathrm{~F})$ semi-rigid ureteroscope alongside a safety guide-wire under vision and we performed fragmentation of encrustation and removed the fragments. We had one patient with heavy encrustations in the ureteric component and associated cardiac disease that made him unfit for anesthesia, he needed ten sessions of ESWL to render him stone- and stent-free. Encrustations with large stone burden on the kidney component of the stent were solved by percutaneous nephrolithotripsy (PCNL). Solving the bladder and ureteric components, always preceded PCNL. Pyelolithotomy was carried out for managing the proximal components in children because we have no pediatric PCNL set in our center and in one adult due to accidental technical problem in our endoscopy unit at that time. We resorted to nephrectomy in two patients who had large bladder and kidney stones burden with loss of kidney function which was demonstrated by radioisotope scanning. Cystolithotomy for the bladder component was done during the same session. All procedures were done without intra-operative complications, and there were no significant postoperative complications. Two cases with totally encrusted ureteric stent and large stone burden over the entire length of the stent worth detailed reporting as they were managed by cystolithotomy, ureterolithotomy and pyelolithotomy in a single anesthetic session. One of them had a solitary ectopic pelvic kidney.

\section{Case 1}

A five year old boy who had a congenital right solitary ectopic pelvic kidney and presented with a ureteral stent retained for about three years which was inserted as an emergency procedure to relieve upper urinary obstruction. Poor compliance was the reason for long indwelling time. His renal functions were normal. KUB and IVU radiographs are shown in (Figures 1,2). Through a single small oblique incision in the right lower abdomen and in a single anesthetic session, cystolithotomy, then ureterolithotomy and pyelolithotomy were performed

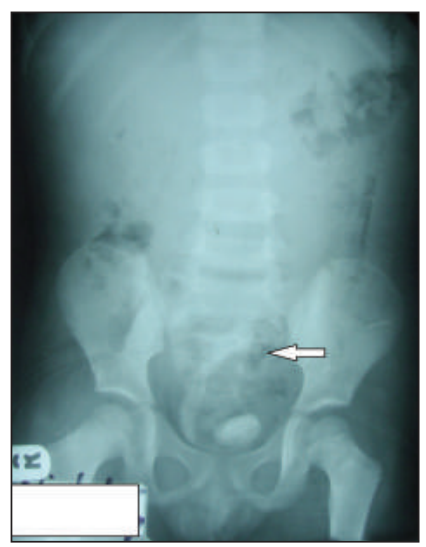

Figure 1.

$K U B X$ ray film shows severe encrustations around ureteral stent, renal pelvis and in the bladder.

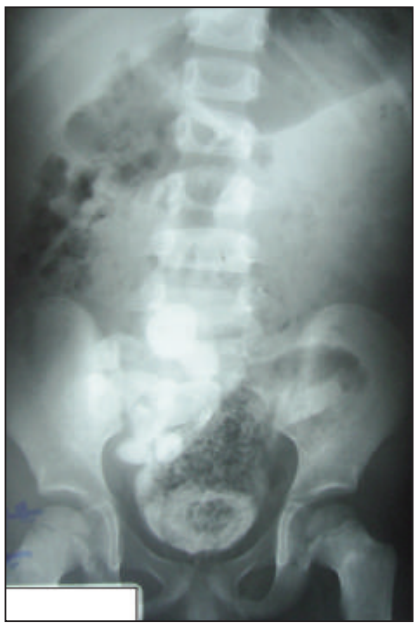

\section{Figure 2.}

Intravenous Urography shows solitary pelvic kidney with significant hydronephrosis.

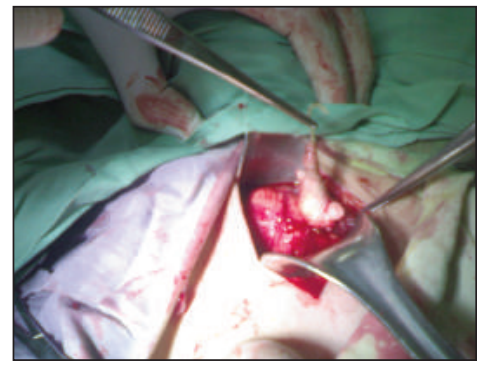

Figure 3.

Oblique lower abdominal incision and removal of the renal pelvic stone through pyelotomy.

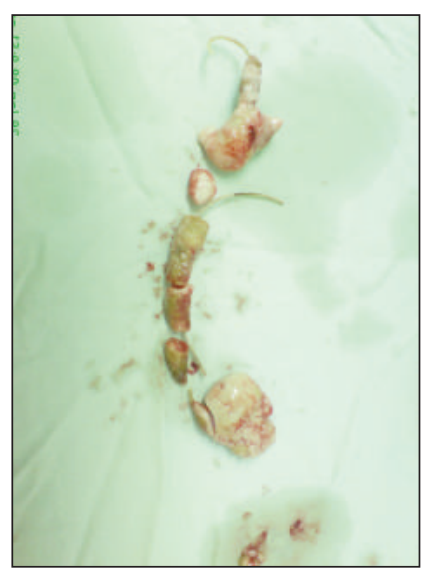

Figure 4.

Demonstration of the stones with the extracted retained ureteral stent after their reconfiguration.

for removal of the stent and stone burden. (Figures 3, 4). Control urinary tract ultrasound showed clearance of stones and the double $\mathrm{j}$ stent removed two weeks later. 


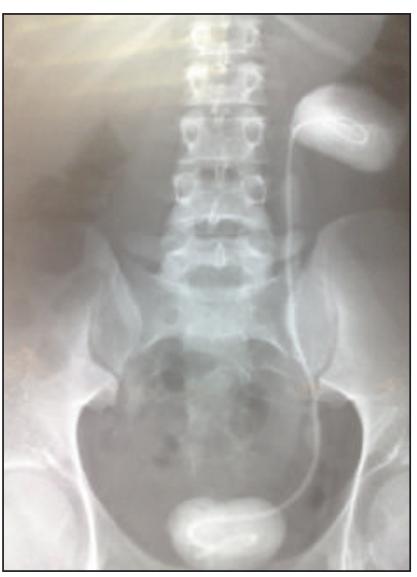

Figure 5.

KUB $X$ ray film shows severe encrustations and large stone burden around retained ureteric stent.

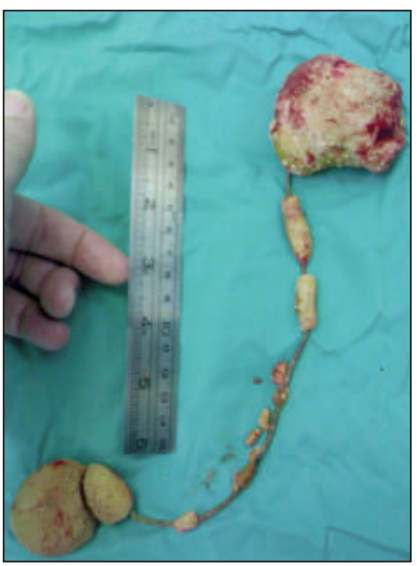

Figure 6.

Shows demonstration of the stones with the extracted retained ureteric stent after their reconfiguration.

\section{Case 2}

This was a 25-year-old man who had a forgotten encrusted ureteral stent placed as an emergency procedure to relieve a ureteric obstruction two years prior to his presentation to our department. Poor compliance was the reason for long indwelling time.

His KUB radiograph is shown in (Figure 5). The vesical and lower ureteric calcifications were managed by cystolithotomy and ureterolithotomy through a lower abdominal incision. The patient was then shifted to flank position where pyelolithotomy and ureterolithotomy for removal of the kidney and upper ureteric encrustations. All of these procedures were performed during a single anesthetic session (Figure 6).

\section{Discussion}

Severely encrusted forgotten ureteral stent is one of the difficult problems in urological practice. Major complications associated with retained stents include infection, migration, fragmentation, stone formation, and ureteral obstruction $(6,7,14-17)$. These complications, in addition to the potential need for multiple surgical interventions and the lack of defined therapeutic guidelines for treatment represent a real challenge for urologist $(3,15$, $16,18)$. Although the exact etiology of encrustation is unclear, the incidence of encrustation increases with indwelling stent duration. In one study, overall $47.0 \%$ of stents were encrusted and the encrustation rate was $26.8 \%$ at less than 6 weeks, $56.9 \%$ at 6 to 12 weeks and $75.9 \%$ at more than 12 weeks (17). Risk factors for stent encrustation are poor patient compliance to follow-up, long indwelling time, sepsis, pyelonephritis, recurrent or residual stone formation, lithogenic history, chronic renal failure, pregnancy metabolic and congenital abnormalities $(2,10,11,18)$. The stent indwelling duration in the current series ranged between 4 months to 16 years, with average of 24.2 months, which is comparable with the mean duration time of 22.7 months reported by Monga et al. (19). Still two other series reported longer average indwelling time of 4.4 years and 4.9 years, with a range of 1-8 years and 1-12 years respectively $(4,7)$. Many times, stents are forgotten either because of illiteracy, non-compliance of the patients who ignore or forget physician advice regarding its timely removal. Poor compliance of the patients was the commonest reason for forgotten stents in our study and this can be explained to some extent by the educational level of patients, as many of our patients were either of low educational level or illiterates. All of the retained stents, except two, were inserted in one city (the capital Sana'a) due lack of endourologic units in the other cities till the near time. As most of patients lived in areas far from the capital Sana'a, they found it difficult to come back for follow-up or removal of their stents. This constitutes another contributing factor for poor compliance and therefore stent missing. The inability of the patient to attend again to hospitals due to financial reasons (poverty) is another considerable cause for retained stent problems in our patients. Delayed referral from ESWL department to the endoscopy department for removal of the stent within the optimum time is a third reason for stent retention. The last important contributing factor for retained stents in our study was the presence of communication gaps between patient and physician, with failure of the physician to adequately council the patient about the presence of stent, and/or the need for its timely removal. Similar to our series, some other series reported poor compliance as the most common reason for retention of these stents $(1,4)$ while delayed referral by lithotripsy department for stent replacement within the optimal time was reported as the commonest reason by another series (2). Multimodal approach is often required for the management of forgotten ureteral stents to achieve successful retrieval of the retained stent and removal of associated stone burden. Although there are no standard and specific guidelines for the management of encrusted ureteral stents, many authors have reported their series and proposed their own management algorithms (1, 3, 7, 11). These include multiple urological modalities which may require single or multiple endourological sessions. Our treatment options were based upon our technical situations and resources. We utilized an average of 2.25 urologic procedures per patient to render our patients stent and stone free, an average that is comparable with the results of other series which reported average of 2.7 and 2.38 procedures for clearing their patients from retained stents and the associated stones $(1,2)$. Bostanci et al. reported the use of various combinations of endourological techniques in 19 patients with encrusted 
ureteral stents and they were able to render all patients stent and stone free after a single anesthetic session with minimal morbidity and short hospital stay (14). Other reports of fewer patients undergoing one-stage removal of encrusted ureteral stents were also published by other authors $(2,15,20)$. In 25 out of 40 of our patients, we were able to remove the retained stents and associated stone burden in a single anesthetic session. We were obligated to perform open pyelolithotomy in children due to unavailability of pediatric PCNL set in our center and in one adult due to accidental technical trouble in our endoscopy unit at that time.

The advent of modern endourologic technology has enabled the removal of all the retained stents utilizing complete endourologic approach. However, in some cases of sever encrustations, endoscopic manipulations may fail and the options of open or laparoscopic surgery are considered $(1,2,7)$.

\section{Conclusions}

The widespread use of indwelling ureteral stent has been translated into documentation of increased number of forgotten stents with their potential complications. Stent encrustation and stone formation are among the serious complications of retained ureteral stents.

Noncompliance of some patients together with poor communication between patients and attending urologists constitute important contributing factors to the missing of ureteral stents.

The retrieval of severely encrusted retained ureteral stent and its associated stone burden poses a real management dilemma for urologists due to the need for multimodal procedures and the lack of standardized treatment plan. Ideally, the best treatment of these difficult problems is prevention through patients' education and proper patient-physician communication to conduct clear information to the patient about the presence of the indwelling stent, the risks associated with prolonged indwelling times and the need for its timely removal or replacement.

\section{REFERENCES}

1. Lam JS, Gupta M. Tips and tricks for the management of retained ureteral stents. J Endourol. 2002; 16:733-41.

2. Rana AM, Sabooh A. Management strategies and results for severely encrusted retained ureteral stents. J Endourol. 2007; 2:62832.

3. Acosta-Miranda AM, Milner J, Turk TM. The FECal Double-J: a simplified approach in the management of encrusted and retained ureteral stents. J Endourol. 2009; 23:409-15.

4. Rabani SM. Combined percutaneous and transurethral lithotripsy for forgotten ureteral stents with giant encrustation. Nephrourol Mon. 2012; 4:633-5.

5. Keane PF, Bonner MC, Johnston SR, et al. Characterization of biofilm and encrustation on ureteric stents in vivo. Br J Urol. 1994; 73:687-91.

6. Mohan-Pillai K, Keeley FX Jr, Moussa SA, et al. Endourological management of severely encrusted ureteral stents. J Endourol. 1999; 13:377-9.
7. Murthy KV, Reddy SJ, Prasad DV. Endourological management of forgotten encrusted ureteral stents. Int Braz J Urol. 2010; 36:420-9.

8. Zimskind PD, Fetter TR, Wilkerson JL. Clinical use of long-term indwelling silicone rubber ureteral splints inserted cystoscopically. $J$ Urol. 1967; 97:840-4.

9. Ather MH, Talati J, Biyabani R. Physician responsibility for removal of implants: the case for a computerized program for tracking overdue double-J stents. Tech Urol. 2000; 6:189-92.

10. Borboroglu PG, Kane CJ. Current management of severely encrusted ureteral stents with a large associated stone burden. J Urol. 2000; 164:648-50.

11. Singh I, Gupta NP, Hemal AK, et al. Severely encrusted polyurethane ureteral stents: management and analysis of potential risk factors. Urology. 2001; 58:526-31.

12. Adanur S1, Ozkaya F. Challenges in treatment and diagnosis of forgotten/encrusted double-J ureteral stents: the largest single-center experience. Ren Fail. 2016; 38:920-6.

13. Singh V, Srinivastava A, Kapoor R, Kumar A. Can the complicated forgotten indwelling ureteric stents be lethal? Int Urol Nephrol. 2005; 37:541-6.

14. Bostanci Y, Ozden E, Atac F, et al. Single session removal of forgotten encrusted ureteral stents: combined endourological approach. Urol Res. 2012; 40:523-9.

15. Singh D, Goel A, Ahmed N, Singh BP. Forgotten stent leading to complex panurinary stone: single-sitting endourologic management. BMJ Case Rep. 2011; 2011:bcr0620103079

16. Ecke TH, Hallmann S, Ruttloff J. Multimodal stone therapy for two forgotten and encrusted ureteral stents: a case report. Cases J. 2009; 2:106.

17. Kawahara $T$, Ito $H$, Terao $H$, et al. Ureteral stent encrustation, incrustation, and coloring: morbidity related to indwelling times. J Endourol. 2012; 26:178-82.

18. Abdelaziz AY, Fouda WB, Mosharafa AA, et al. Forgotten ureteral stents: Risk factors, complications and management. AFJU 2018; 24:28-33.

19. Monga M, Klein E, Castañeda-Zúñiga WR, Thomas R. The forgotten indwelling ureteral stent: a urological dilemma. J Urol. 1995; 153:1817-9.

20. Bukkapatnam R, Seigne J, Helal M. 1-step removal of encrusted retained ureteral stents. J Urol. 2003; 170:1111-4.

\section{Correspondence}

Ibrahim Ahmed Alnadhari, MD (Corresponding Author)

ibrahimah1978@yahoo.com

Morshed Ali Salah, MD

morshed.salah@gmail.com

Urology and Nephrology Center, at Al-Thawra Modern General

and Teaching Hospital, Sana'a, Yemen

Department of Urology, Al Wakra Hospital, Hamad Medical Corporation, Al Wakra, Qatar

Mohammed Ahmed Alwan, MD

MAlwan197200@gmail.com

Abdulelah M Ghilan, MD

dr_ghilan1@yahoo.com

Urology and Nephrology Center, at Al-Thawra Modern General

and Teaching Hospital, Sana'a, Yemen, AlKhamseen Street, Sana'a, Yemen 